\title{
Application of instantaneous phase detection technology based on laser displacement sensor in fault diagnosis of spur gear of rotation vector reducer
}

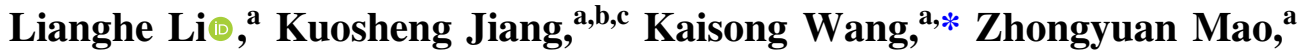 \\ Zaichuan Fan, ${ }^{a}$ and Jiashu Hou ${ }^{a}$ \\ ${ }^{a}$ Anhui University of Science and Technology, School of Mechanical Engineering, \\ Anhui, China \\ ${ }^{b}$ Anhui University of Science and Technology, State Key Laboratory of Mining Response \\ and Disaster Prevention and Control in Deep Coal Mines, Huainan, China \\ ${ }^{\mathrm{c} S}$ Shaanxi Automobile Holding Group Huainan Special Purpose Vehicle Co., Ltd., \\ Huainan, China
}

\begin{abstract}
Aiming at the problem of a spur gear fault of the rotation vector (RV) reducer in industrial robots, an instantaneous phase detection technology based on eccentric modulation and laser displacement sensor is proposed. Taking the RV-20E reducer as the object, through the analysis of the RV reducer gear fault mechanism, an RV reducer fault diagnosis test bed is developed to simulate the actual operating conditions of the RV reducer of industrial robot. The instantaneous phase information of the RV reducer is collected, and the adaptive permutation entropy is used to process the instantaneous phase data, which can judge the gear fault type quickly and effectively. The test results show that the fault diagnosis test bench based on this detection technology can effectively and quantitatively judge the fault type of the spur gear of the RV reducer. $\odot$ The Authors. Published by SPIE under a Creative Commons Attribution 4.0 International License. Distribution or reproduction of this work in whole or in part requires full attribution of the original publication, including its DOI. [DOI: 10.1117/1.OE.61.6.061411]
\end{abstract}

Keywords: rotate vector reducer; gear failure; instantaneous phase; adaptive permutation entropy; quantitative diagnosis.

Paper 20211413SS received Dec. 3, 2021; accepted for publication Feb. 2, 2022; published online Feb. 22, 2022.

\section{Introduction}

Industrial robots have become an indispensable part of the manufacturing industry, aerospace, and other fields. The ownership and automation level of industrial robots are important indicators to measure the level of national technological innovation and high-end manufacturing. The RV reducer is the precise core component of industrial robots that is used in the base, arm, shoulder, and other joint positions. The RV reducer is also an important guarantee for the rapid and accurate execution of various complex mechanical actions, and it is also a key factor affecting the smoothness of the robot's motion.

In recent years, high-precision laser displacement sensors have been widely used in various situations. Bi et al. ${ }^{1}$ proposed an all-fiber tunable distributed Bragg reflector laser rangefinder, which uses self-mixing effect to achieve high-precision absolute distance measurement in the range of 1.88 to $3.33 \mathrm{~m}$. Zhang et al. ${ }^{2}$ proposed a laser Doppler angular vimeter using $\mathrm{He}-\mathrm{Ne}$ dual-frequency ring laser. The angular vibration displacement can be measured by detecting the interference signal of the beam diffracted by the two grating surfaces. Bohkman et al. ${ }^{3}$ proposed an implementation method of dynamic laser goniometer (LG) for non-contact measurement of angular position of objects. At the same time, many experts and scholars have carried out a series of studies on the comprehensive performance of the RV reducer, mainly from the RV reducer transmission accuracy, torsional stiffness, and other aspects. ${ }^{4-7}$ Wang et al. ${ }^{8}$ established an improved cycloidal gear meshing efficiency model by using the numerical analysis method

*Address all correspondence to Kaisong Wang, 328803633@qq.com 
of mixed elastohydrodynamic lubrication. Yang et al. ${ }^{9}$ proposed an analytical error modeling method for RV reducer's overconstrained structure, and clearly established the relationship between the original error and transmission accuracy. Chen et al. ${ }^{10}$ proposed a fault diagnosis method for RV reducer based on nonlinear output frequency-response function and deep convolutional neural network. Rohan et al. ${ }^{11}$ proposed to use the embedded current signal of the control unit as a method to detect and diagnose mechanical faults. A batch of new diagnostic techniques is proposed for gear faults. ${ }^{12-15}$ Pan et al. ${ }^{16}$ proposed a new signal decomposition algorithm, nonlinear sparse mode decomposition, for the analysis of the RV reducer gearbox fault signals. Yu and Zhou ${ }^{15}$ proposed a new deep neural networks (DNN), namely onedimensional residual convolution autoencoder (1-DRCAE), for learning unsupervised learning features directly from vibration signals. Schmidt et al. ${ }^{17}$ proposed a system framework for obtaining consistent feature surfaces under time-varying operating conditions, which is suitable for fault diagnosis of gears and bearings under time-varying operating conditions. Jiang et al. ${ }^{18}$ propose a novel ascension multi-wavelet method for diagnosing the undergoing degradation state and predicting the remaining useful life of the bearings.

This article takes the RV-20E reducer as the research object, and conducts research on the failure of the spur gear during the transmission of the reducer. The instantaneous phase information of the RV reducer is obtained based on laser displacement sensor and eccentric wheel. By processing the instantaneous phase information, the fault type of spur gear can be judged, which provides reference for the fault diagnosis of the spur gear of the RV reducer.

\section{RV Reducer Spur Gear Failure Mechanism Analysis}

\subsection{Composition Structure and Transmission Mechanism of the RV Reducer}

The composition of the RV reducer is compact and complex. It is mainly composed of housing, input gear shaft, spur gear, planet carrier, output disk, cycloid, crank shaft, needle roller, dust retaining ring, main bearing, tapered roller bearings, needle roller bearings, spring retainers, and other parts, Fig. 1 shows the explosion diagram of structure composition of the RV-20E-121 reducer.

$\mathrm{RV}$ reducer is a two-stage reduction mechanism, which includes planetary gear transmission and cycloid pin wheel transmission. The transmission process includes four parts: input gear shaft, involute planetary gear transmission, cycloid pin wheel transmission, output disc output, or needle gear shell output. The transmission schematic diagram is shown in Fig. 2.

\subsection{Gear Vibration Mechanism}

Under normal circumstances, when studying the vibration mechanism of gears, the gear vibration system is often simplified to analyze its meshing vibration. The simplified model of gear meshing vibration is shown in Fig. 3.

According to the vibration model of gear meshing shown in Fig. 3, the dynamic equation at the meshing point can be obtained as

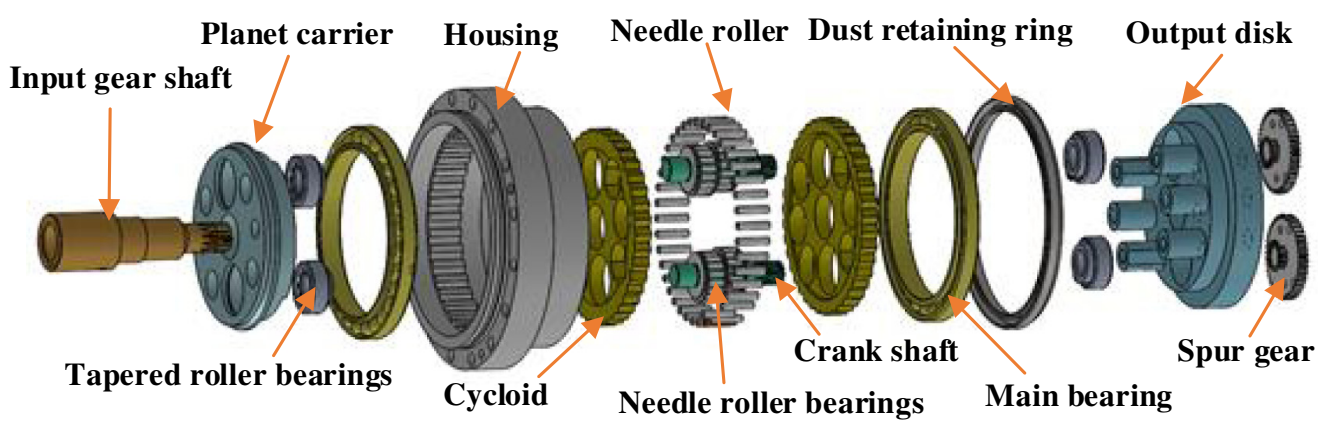

Fig. 1 Explosion diagram of structure composition of the RV-20E-121 reducer. 


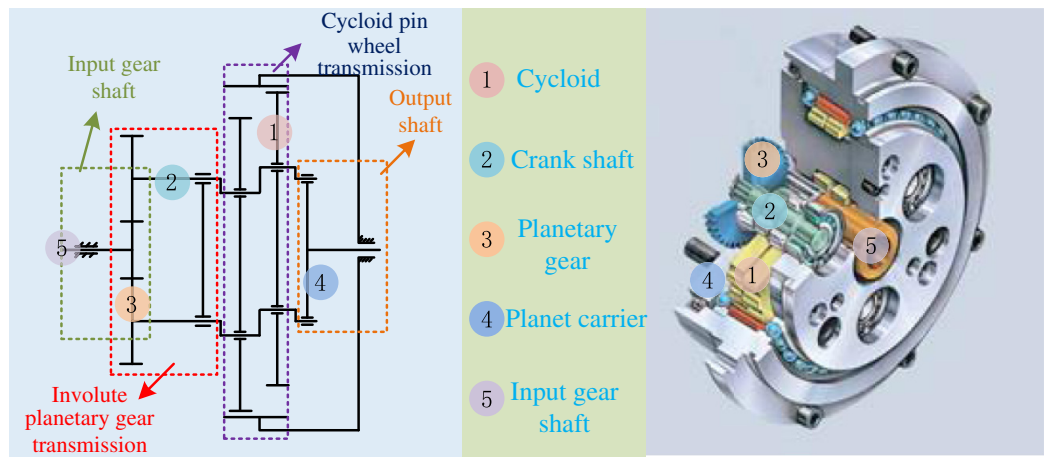

Fig. 2 RV reducer transmission schematic diagram.

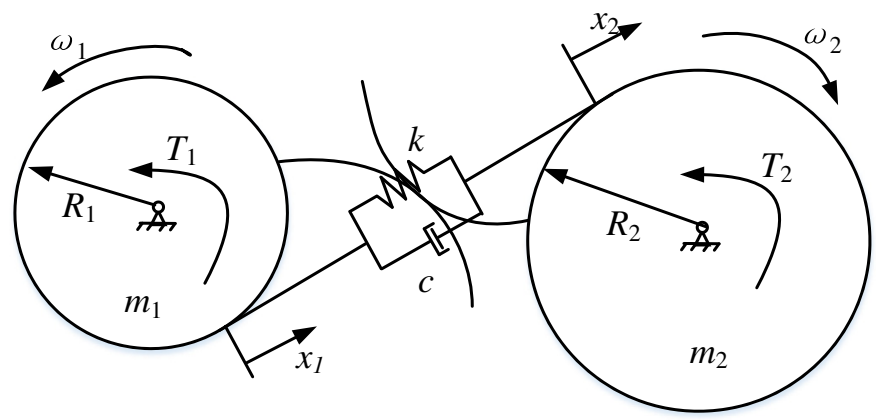

Fig. 3 Simplified model of gear meshing vibration.

$$
M \ddot{x}+c \dot{x}+k x=F
$$

In the formula, $F$ is the external excitation to the system; $k$ is the equivalent stiffness of the gear pair meshing; $c$ is the equivalent damping of the gear pair meshing; $M$ is the equivalent mass of the gear pair, $M=\left(m_{1} m_{2}\right) /\left(m_{1}+m_{2}\right) ; x$ is the relative displacement in the meshing direction, $x=x_{1}-x_{2}$

$$
M \ddot{x}+c \dot{x}+k x=k E_{1}+k E_{2} .
$$

In the formula, $E_{1}$ is the average static elastic deformation; $E_{2}$ is the relative displacement function between the two teeth caused by error or failure; $k E_{1}$ is the vibration of the gear under normal conditions; $k E_{2}$ mainly represents the side frequency signal that will appear in the signal when the fault occurs, and the relationship between gear stiffness $k$ and relative displacement function $E_{2}$ and failure.

\subsection{Analysis of the Fault Signal of the Gear}

The mathematical model of the gear fault characteristic signal can be expressed as

$$
x(t)=\sum_{n=0}^{N} A_{n}\left[1+a_{n}(t)\right] \cos \left[2 \pi n f_{s} t+\phi_{n}+b_{n}(t)\right]+\left(1+\cos \left(2 \pi n f_{g} t\right)\right) \cos \left(2 \pi n f_{g r} t\right) .
$$

In the formula, $a_{n}(t)$ and $b_{n}(t)$ represent the amplitude and phase modulation function of the harmonic component of the $n$ 'th meshing frequency, respectively.

The gear tooth with fault rotates once with the shaft, and the gear tooth meshing defect passes once. The dimensional modulation functions $a_{n}(t)$ and $b_{n}(t)$ are functions of the gear rotation frequency, which can be expressed as 
Li et al.: Application of instantaneous phase detection technology based on laser displacement sensor...

$$
\begin{gathered}
a_{n}(t)=\sum_{k=0}^{K} B_{n, k} \cos \left(2 \pi n f_{g} t+\varphi_{n, k}\right), \\
b_{n}(t)=\sum_{k=0}^{K} C_{n, k} \cos \left(2 \pi n f_{g} t+\varphi_{n, k}\right) \bar{\varphi}_{n, k},
\end{gathered}
$$

where $B_{n, k}$ is the $k^{\prime}$ th order component amplitude modulation coefficient; $\varphi_{n, k}$ is the initial phase of the $k^{\prime}$ th order component amplitude modulation function; $C_{n, k}$ is the $k^{\prime}$ th order component phase modulation coefficient; $\bar{\varphi}_{n, k}$ is the initial phase of the $k^{\prime}$ th order component phase modulation function; $f_{g}$ is the gear meshing frequency; and $f_{g r}$ is the gear meshing resonance frequency.

\section{Introduction of Instantaneous Phase Detection Technology Based on Eccentric Modulation}

The instantaneous phase signal contains a wealth of information about the state of machinery health, and the change law of instantaneous phase contains a wealth of signal characteristics, it plays an important role in rotating machinery fault diagnosis, rotor dynamic balance detection, and RV reducer performance evaluation. This paper adopts an instantaneous phase detection method based on the principle of eccentric modulation. This method is derived from the kinematic law between the cam and the push rod. The laser displacement sensor is used to replace the push rod, and the eccentric wheel replaces the cam to realize non-contact measurement. The instantaneous phase is modulated by the eccentric wheel, and the trigonometric basis function is used to demodulate the signal containing the fault characteristics.

\subsection{Instantaneous Phase Eccentric Modulation}

The instantaneous phase detection technology based on eccentric modulation is derived from the kinematic law between the cam and the push rod. The evolution of the measurement principle is shown in Fig. 4. A laser displacement sensor is used instead of a push rod to achieve non-contact measurement, an eccentric wheel is used to modulate the instantaneous phase, and then a simple trigonometric function is used to demodulate the signal with fault characteristics.

\subsection{Instantaneous Phase Eccentric Demodulation}

The instantaneous phase information can accurately locate the defect position and realize the one-to-one correspondence between the instantaneous phase signal of the same period and the defect position of the slewing ring. Therefore, the accurate extraction of the instantaneous phase

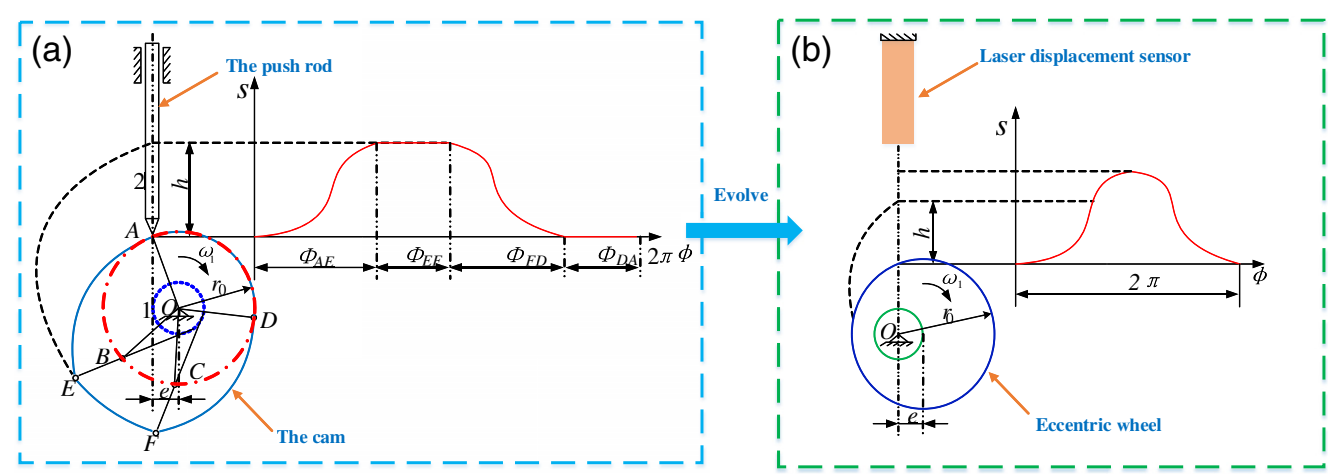

Fig. 4 Evolution of measurement principle. (a) The kinematic law between cam and push rod; (b) transient phase evolution process. 


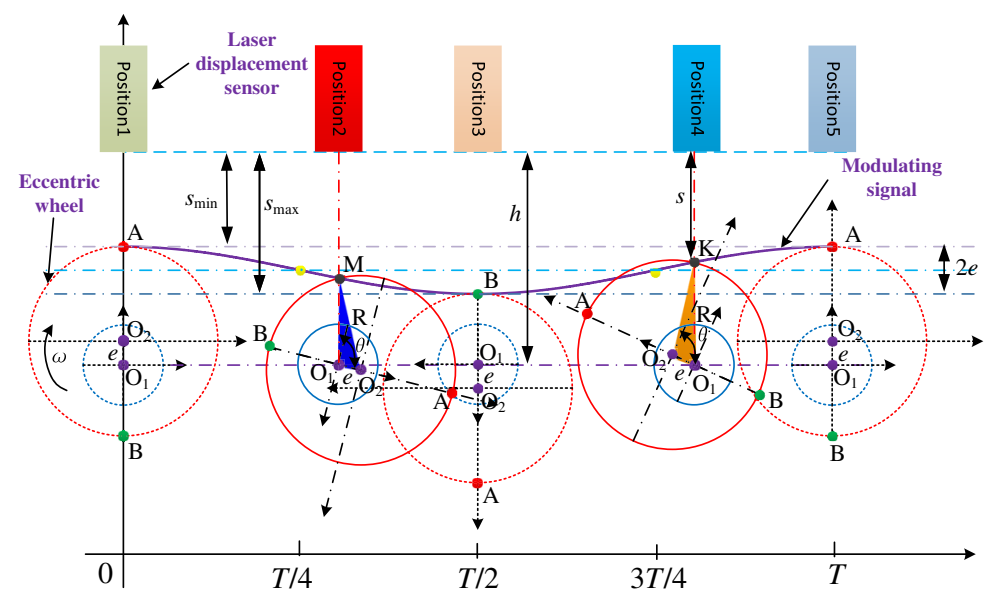

Fig. 5 Schematic diagram of instantaneous phase detection based on eccentric modulation principle.

information is an important factor for the accurate extraction of the defect feature information. The schematic diagram of the instantaneous phase detection technology based on the principle of eccentric modulation is shown in Fig. 5.

From the principle diagram of the instantaneous phase measurement, point $O_{1}$ is the center of rotation of the eccentric wheel, and point $\mathrm{O}_{2}$ is the center of the outer circle of the eccentric wheel. $e$ is the eccentricity of the eccentric wheel. Points A and $\mathrm{B}$ in the figure are the highest point and the lowest point of the eccentric wheel respectively, which correspond to the minimum and maximum values $S_{\min }$ and $S_{\max }$ of the displacement measured by the laser sensor. $\theta$ is the eccentric sleeve based on the $y$ axis rotation angle. When the spindle is running at the speed of $\omega$, the displacement measured by the laser displacement sensor is $S$. The measured displacement $S$ can be converted into the phase $\theta$ through the simple mathematical geometric relationship of $\Delta O_{1} O_{2} M$, and the conversion relationship is shown in Eqs. (6)-(8).

$$
\begin{gathered}
r^{2}=(h-s)^{2}+e^{2}-2(h-s) e \cos \theta, \\
\theta=\arccos \left\{\left[(h-s)^{2}+e^{2}-r^{2}\right] / 2 e(h-s)\right\}, \\
h=r+e+s_{\min },
\end{gathered}
$$

where $h$ is the distance between the probe of the laser displacement sensor and the axis of the spindle, and $r=d / 2$ is the radius of the outer circle of the eccentric wheel.

In this paper, Panasonic HL-G103-S-J laser displacement sensor is selected, its measurement range is $\pm 4 \mathrm{~mm}$, the resolution $(\varepsilon)$ is $0.5 \mu \mathrm{m}$, self-made eccentric sleeve eccentricity $(e)$ is $4 \mathrm{~mm}$, the eccentric stroke is $2 e=8 \mathrm{~mm}$. The maximum resolution and maximum accuracy of the instantaneous phase detection device developed in this paper are shown in Eqs. (9) and $(10)$

$$
\begin{gathered}
\delta=\frac{2 e}{\varepsilon}=\frac{8 \times 10^{3}}{0.5}=16,000, \\
\sigma=\frac{360 \mathrm{deg}}{\delta}=\frac{360 \mathrm{deg}}{16,000}=0.0225 \mathrm{deg},
\end{gathered}
$$

where $\delta$ is the measurement accuracy of instantaneous phase detection transpose and $\sigma$ is the measurement resolution of the instantaneous phase detection transpose. 


\section{Research on Fault Diagnosis of Spur Gear of the RV Reducer Based on Adaptive Permutation Entropy}

\subsection{RV Reducer Spur Gear Fault Diagnosis Test Bench Construction}

The fault diagnosis test platform for the basic components of the RV reducer designed and built in this paper is mainly composed of driving devices (including servo motors and their controllers), loading devices (including AC motors and their controllers), and detection devices (including eccentric shafts, laser displacement sensors), the measured object (including the spur gear of the RV-20E-121 reducer) and the data acquisition system (including the PC terminal program design and the NI data acquisition card) are composed of five parts, and its basic structure is shown in Fig. 6.

\subsection{Introduction to Adaptive Permutation Entropy}

To realize the quantitative diagnosis of the fault degree of the basic components of the RV reducer, it is necessary to extract the fault characteristic signals of the basic components from the collected signals. To facilitate the later data processing and comparative analysis, the signal amplitude is normalized first, and then the ensemble empirical mode decomposition (EEMD) method is used to extract the fault characteristic signal of the gear. Permutation entropy is used to evaluate the complexity of gears with different failures and to classify bearings and gears with different quality problems. Since the embedding dimension and delay time have a great influence on the permutation entropy, artificial selection is easy to cause errors, and the best effect of phase space reconstruction cannot be achieved. Therefore, the average mutual information (AMI) is used to estimate the delay time of the phase space reconstruction, and the pseudo nearest neighbor (PNN) algorithm is used to estimate the embedding dimension of the phase space reconstruction to realize the adaptive selection of permutation entropy parameters. Finally, a batch experiment is carried out to solve the permutation entropy value.

The delay of phase space reconstruction was estimated using AMI. For reconstruction, the time delay is set to the first local minimum of the AMI. The calculation formula of AMI is as follows:

$$
\operatorname{AMI}(T)=\sum_{i=1}^{N} p\left(x_{i}, x_{i+T}\right) \log _{2}\left[\frac{\left[p\left(x_{i}, x_{i+T}\right)\right]}{p\left(x_{i}\right) p\left(x_{i+T}\right)}\right]
$$

where $N$ is the length of the time series, $p\left(x_{i}\right)=\frac{\left|x_{i}\right|}{N}, p\left(x_{i}, x_{i+T}\right)=\frac{\left|x_{i} \cap x_{i+T}\right|}{N}, T=1$ :Maxlag.

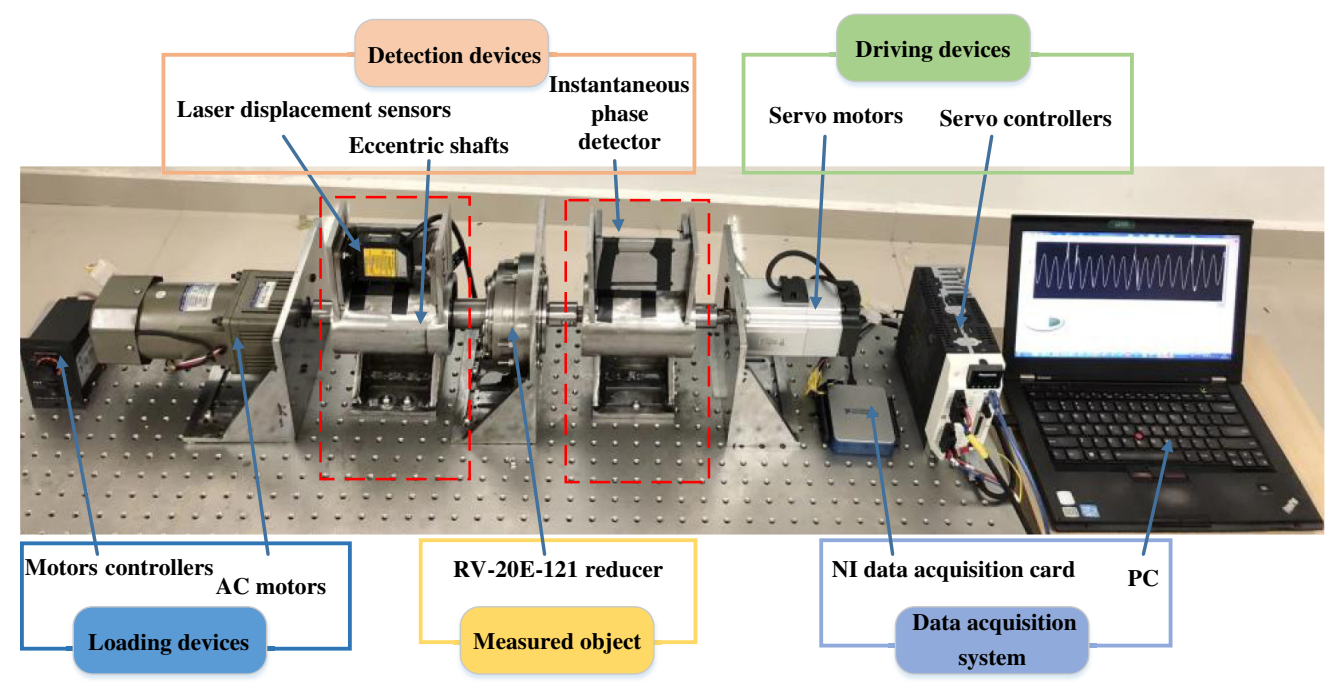

Fig. 6 RV reducer base component fault diagnosis test bed. 
Li et al.: Application of instantaneous phase detection technology based on laser displacement sensor...

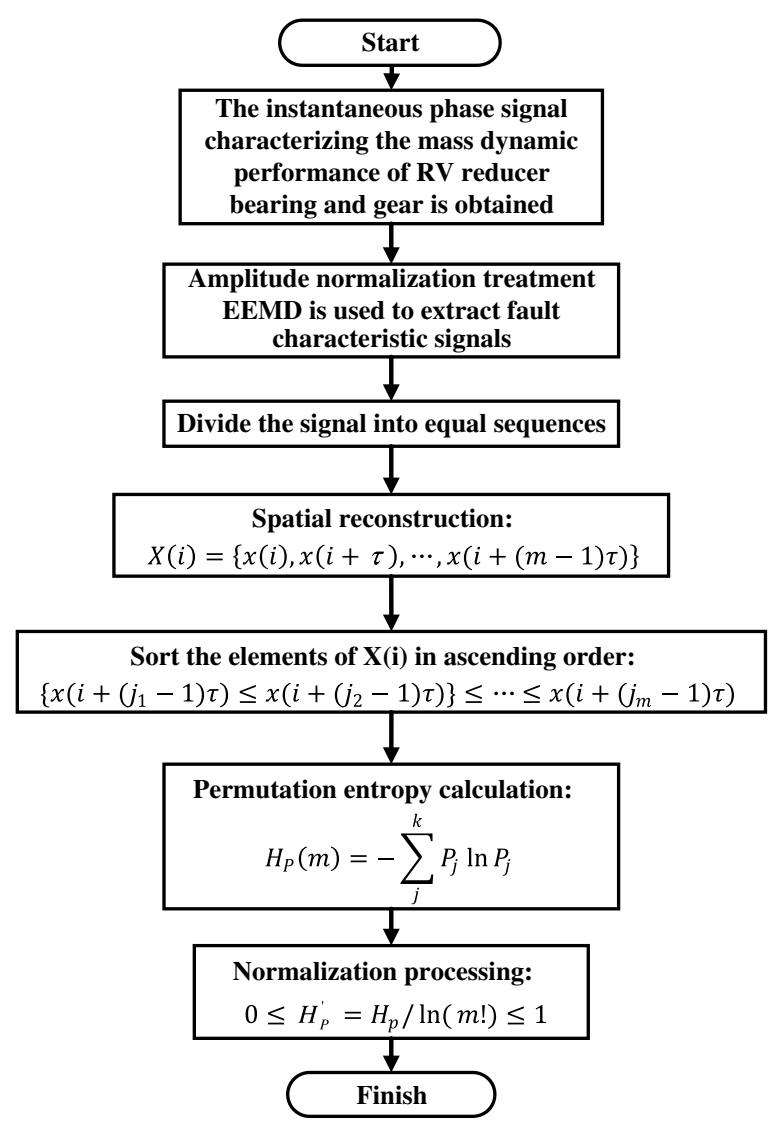

Fig. 7 Calculation steps of permutation entropy.

The embedding dimension of phase space reconstruction is estimated using PNN. If point $X_{i}^{r^{*}}$ and its nearest point $\left\{X_{i}^{r^{*}}\right\}$ in the reconstructed phase space $X_{i}^{r}$ are false neighbors, $i=1: N$, then

$$
\sqrt{\frac{R_{i}^{2}(m+1)-R_{i}^{2}(m)}{R_{i}^{2}(m)}}>D T
$$

where $R_{i}^{2}(m)=\left\|X_{i}^{r}-X_{i}^{r^{*}}\right\|^{2}$ is the distance indicator, $D T$ is the distance threshold.

The main calculation steps of the RV reducer spur gear fault diagnosis and evaluation method based on adaptive permutation entropy are shown in Fig. 7.

\subsection{Spur Gear Fault Diagnosis Test}

The spur gears in the planetary gear transmission mechanism of the RV reducer are set with broken teeth and tooth surface wear faults, respectively. The actual picture of the experimental gear of the RV reducer is shown in Fig. 8. There are three types of RV reducers in the thesis experiment: normal RV reducers, RV reducers with broken spur gears, and RV reducers with worn spur gears. For each type of fault RV reducer, except for the single-source fault of the gear, the other basic components are in normal performance.

The reduction ratio $i=121$ of the RV-20E-121 type reducer, the input shaft speed of the RV reducer is $800 \mathrm{r} / \mathrm{min}$ and the output shaft speed is $6.6 \mathrm{r} / \mathrm{min}$. The voltage signal output by the laser displacement sensor is converted into a displacement signal, and the amplitude is normalized. The time-domain waveforms of the collected normal gear, broken tooth, and tooth surface wear are shown in Fig. 9. 


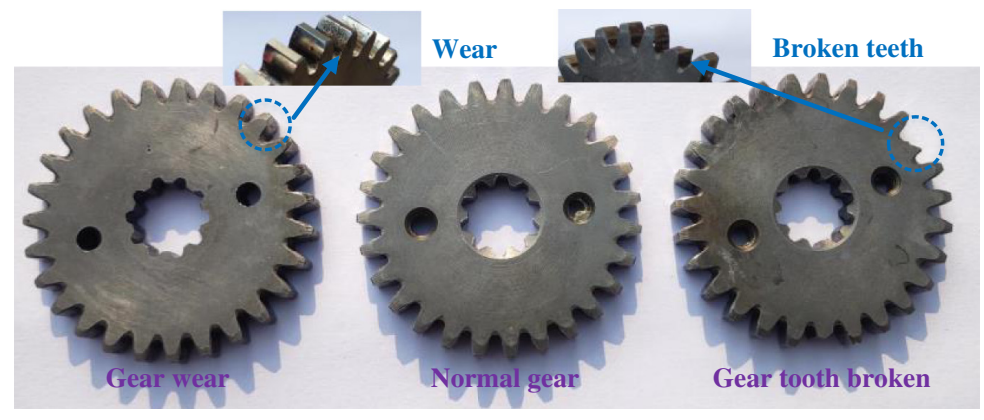

Fig. 8 Experimental gear diagram.
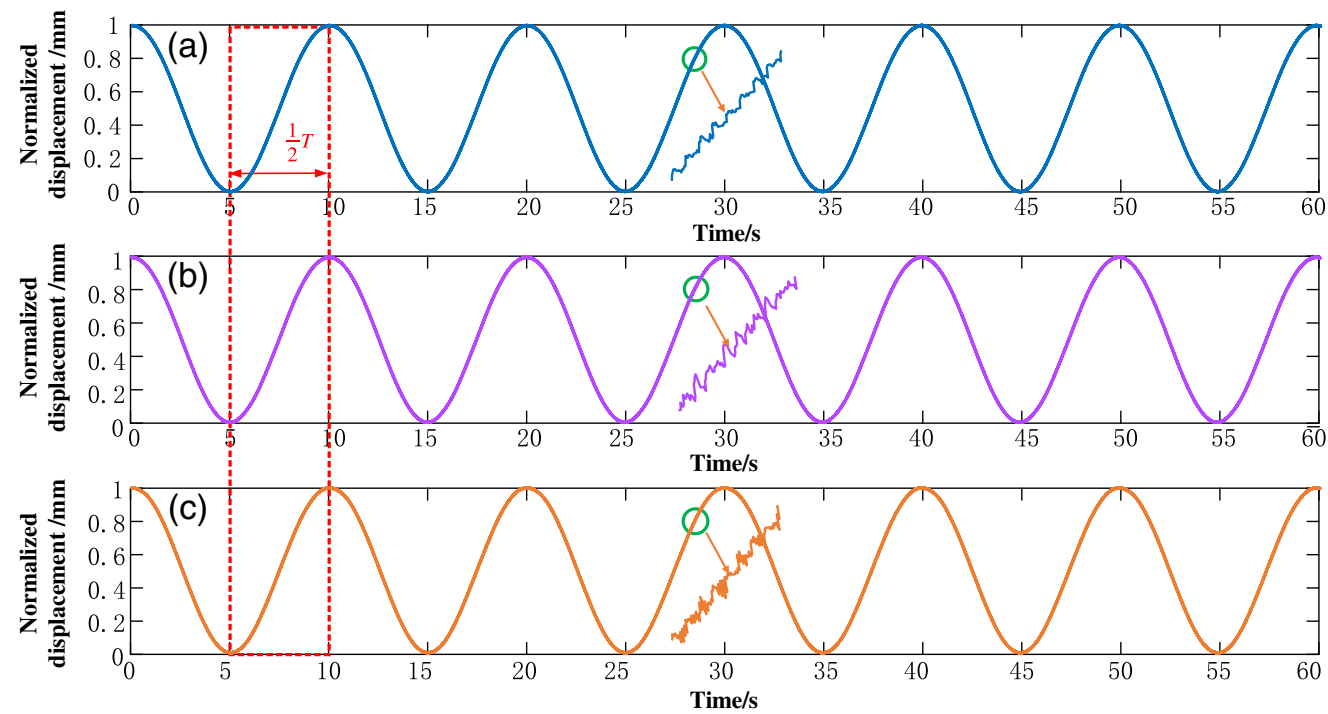

Fig. 9 Time domain signal of gear. (a) Time domain signal of normal gear; (b) time domain signal of gear broken teeth; and (c) time domain signal of gear wear.

Local amplification of the three signals shows that the waveform forms and signal complexity are quite different. Trigonometric function is used to demodulate the three signals to obtain instantaneous phase signals, as shown in Fig. 10. It can be seen from the figure that the instantaneous phase signal changes periodically, and its period is the same as that of the displacement signal, i.e., the time for the eccentric to rotate once. The local details of the three instantaneous phase signals also show the difference of the signal's local characteristics and waveform shape. Both laser displacement signals and instantaneous phase signals contain abundant fault characteristic signals. It can be seen from the time domain waveform detail diagram that the waveform forms of normal gear and different faulty gear are different, and the complexity of signal sequence is also different.

To study the fault transmission mechanism of the RV reducer basic components and collect the signal of output end in one cycle, then the input end needs to run for 121 cycles, i.e., the signal of output end in one cycle already contains enough gear fault characteristics. To facilitate the research and data analysis, the signal of output shaft in half a cycle is selected for analysis. Because the spur gear is in the first level reduction transmission of the RV reducer, after analysis, the output shaft runs for half a cycle, the input shaft needs to run for 61 cycles, and the gear shaft and spur gear engage 20 times at the fault place, so the output shaft signal of $1 / 2 T$ cycle already contains enough fault characteristics. The time domain signal of $1 / 2 T$ period is intercepted for the displacement signal and instantaneous phase signal of each signal at the position corresponding to Figs. 9 and 10. Time domain signals and local details of gear 1/2T laser displacement are 

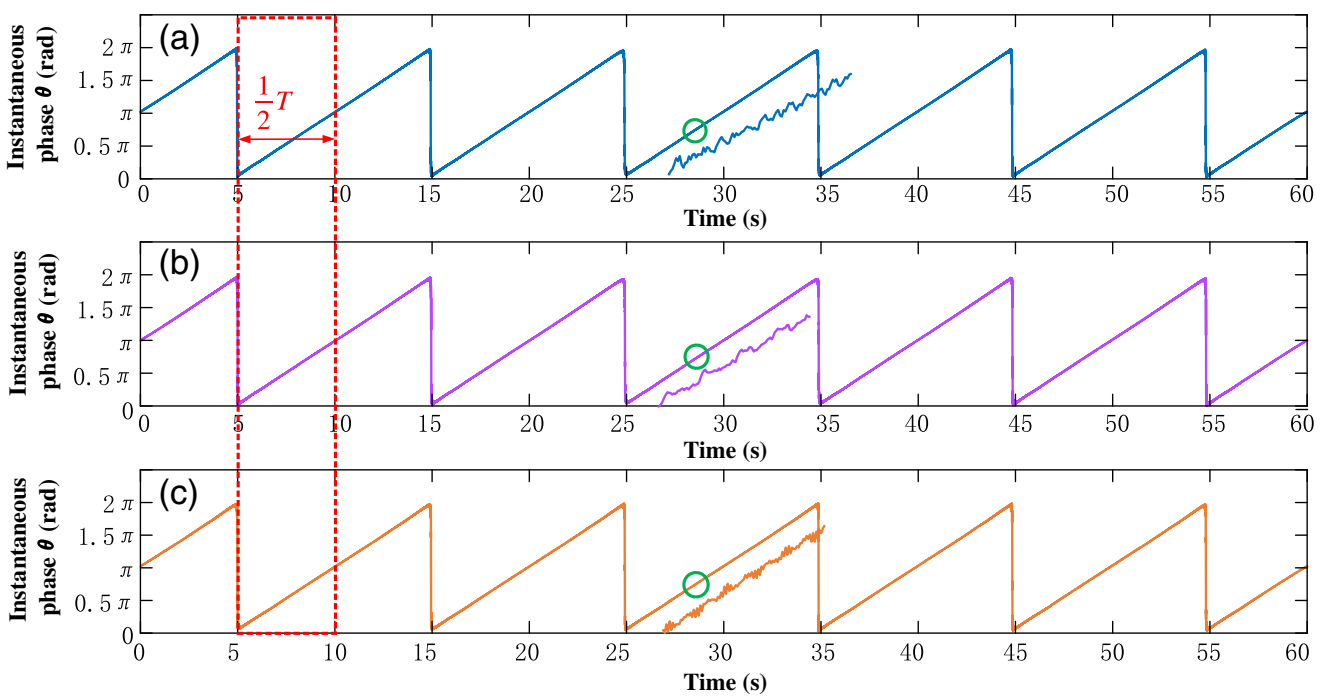

Fig. 10 Instantaneous phase signal of gear. (a) Instantaneous phase signal of normal gear; (b) instantaneous phase signal of gear tooth break; and (c) instantaneous phase signal of gear wear.

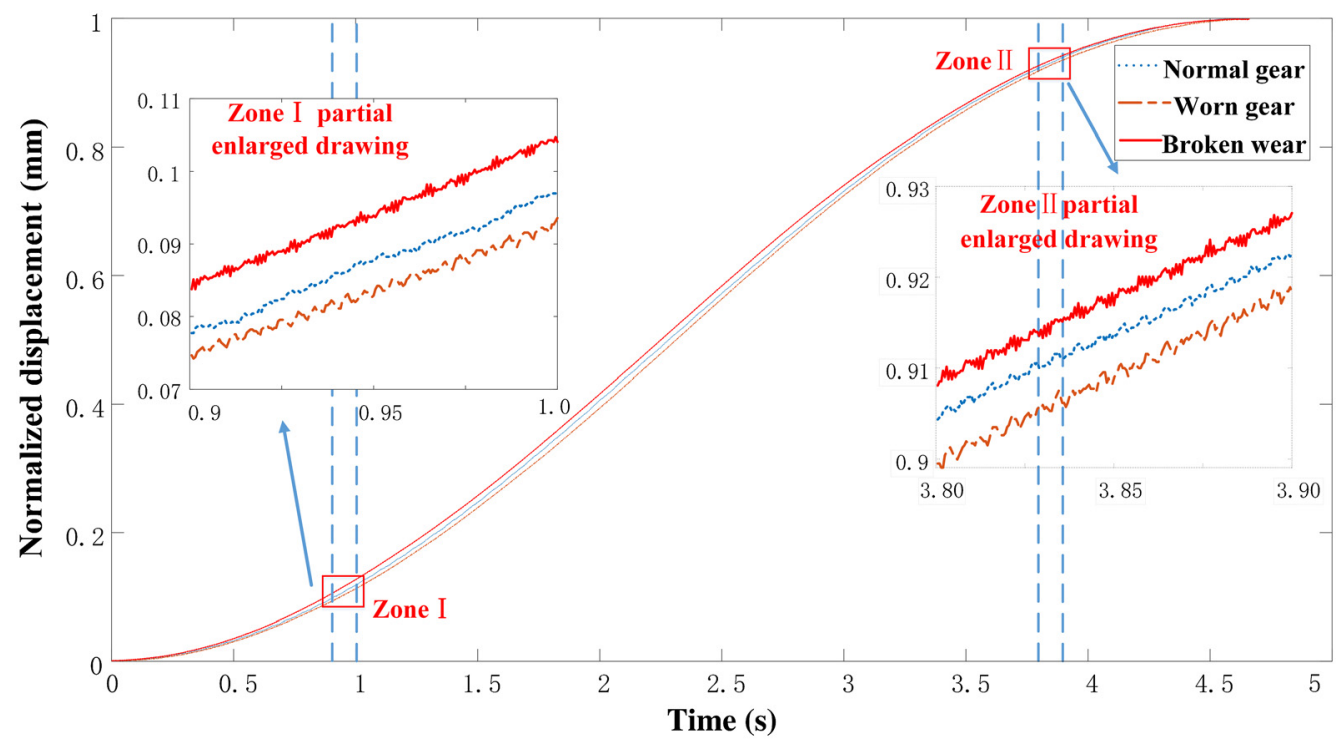

Fig. 11 Time domain signal and local detail diagram of gear.

shown in Fig. 11. Instantaneous phase signal and local detail of gear 1/2T are shown in Fig. 12. It can be clearly seen from the figure that there are differences in time domain signal waveforms of normal gear, worn gear, and broken gear, and the signal waveforms have an upward trend. Because the collected signal is made into a cosine-like signal by the eccentric, its trend is the trend of the modulation signal.

To solve the above phenomenon, EEMD was used to decompose and reconstruct the instantaneous phase signals of normal gear, worn gear, and broken gear respectively, and the gear fault characteristic signals were extracted. It can be seen from the figure that the intrinsic mode function IMF7 is the trend of the original signal. Remove IMF7 and reconstruct other components to obtain a new instantaneous phase reconstruction signal. Figure 13 shows instantaneous phase signal decomposition and signal recomposition of normal gear in section II. Figure 14 shows instantaneous phase signal decomposition and signal recomposition of section II gear with broken teeth. Figure 15 shows instantaneous phase signal decomposition and signal recomposition 
Li et al.: Application of instantaneous phase detection technology based on laser displacement sensor...

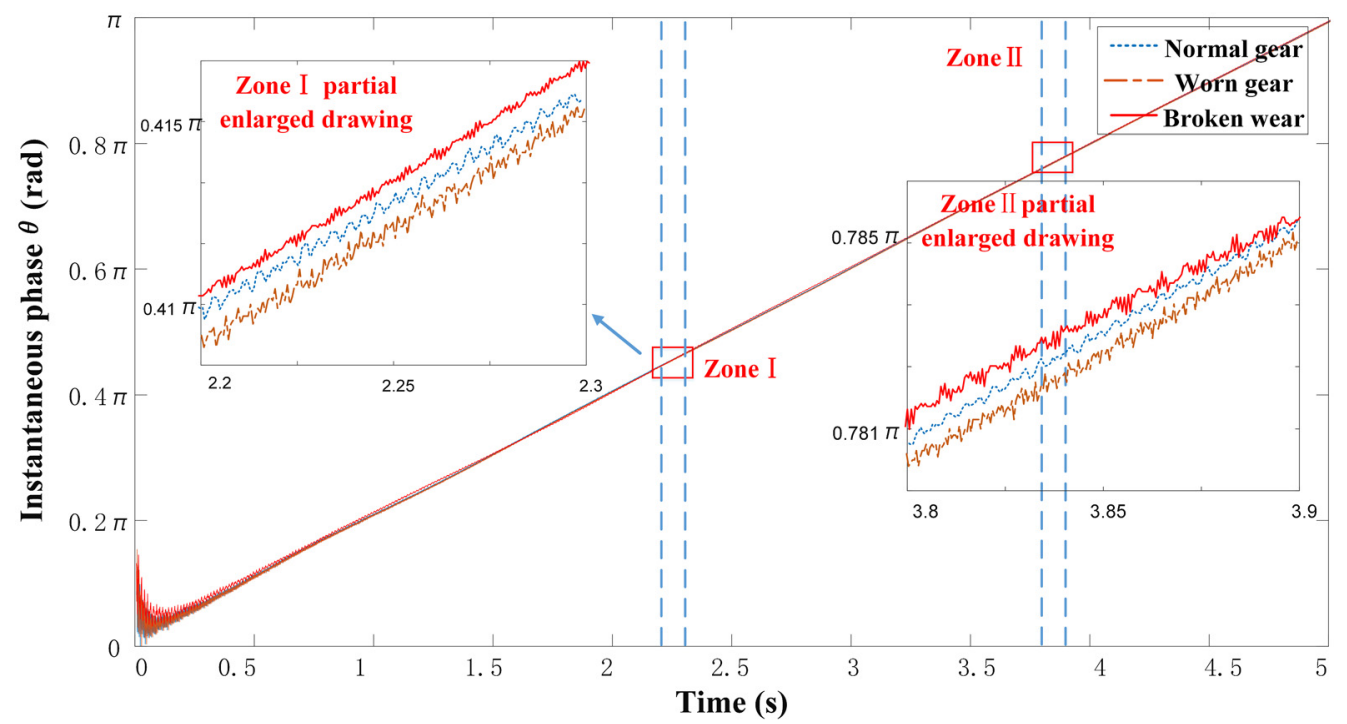

Fig. 12 Instantaneous phase signal and local detail diagram of gear.
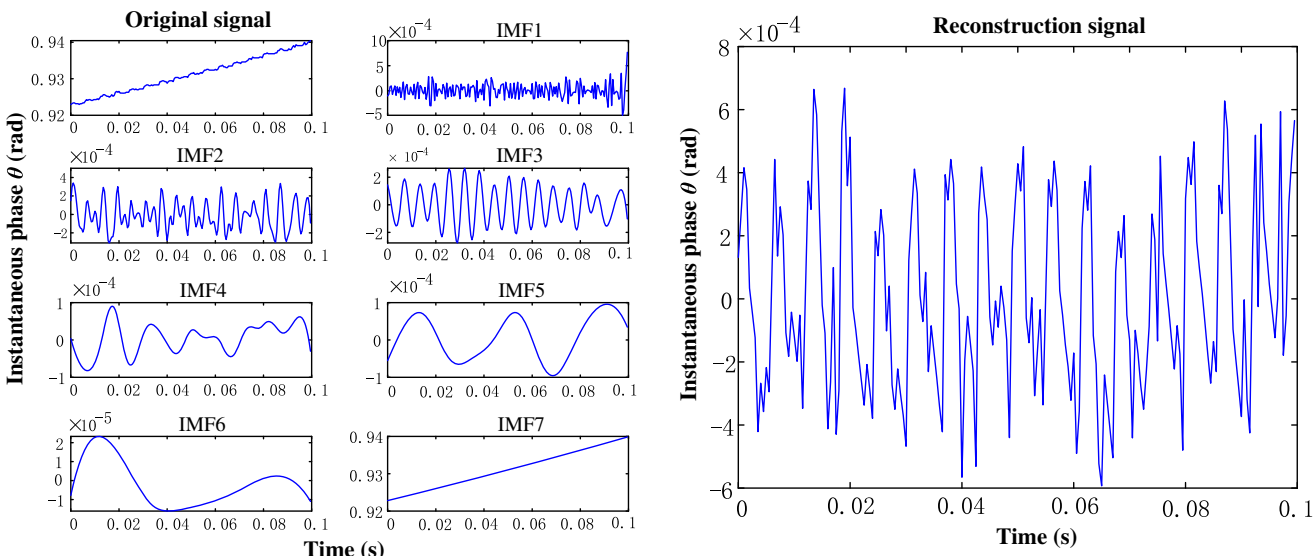

Fig. 13 Section II normal gear instantaneous phase signal decomposition and composition.
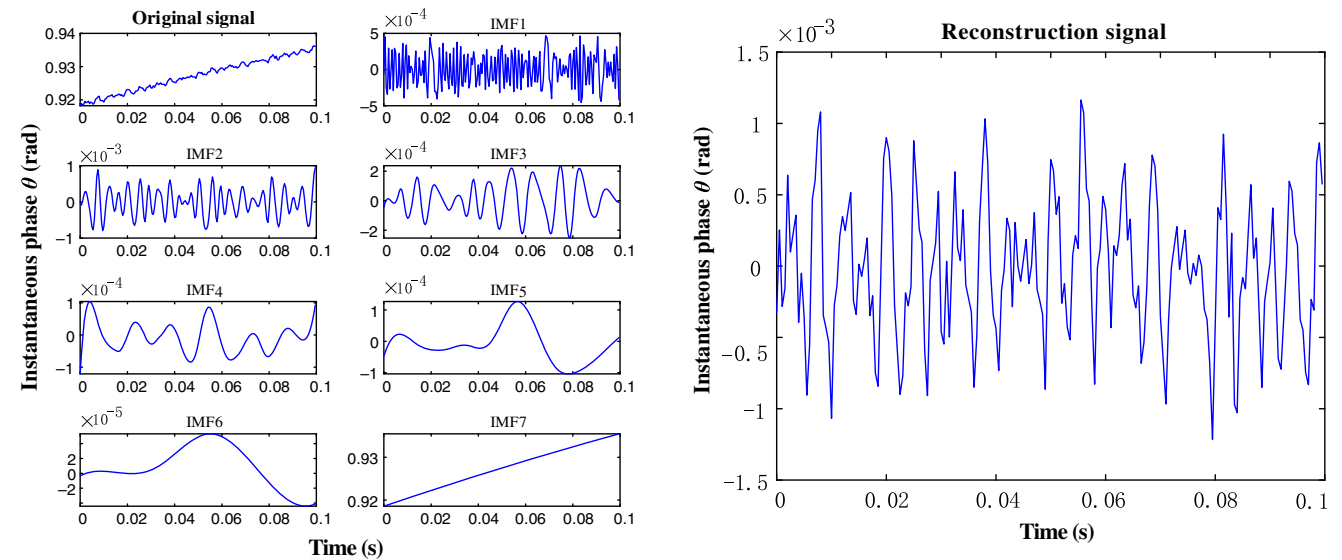

Fig. 14 Section II gear tooth broke instantaneous phase signal decomposition and composition. 
Li et al.: Application of instantaneous phase detection technology based on laser displacement sensor...
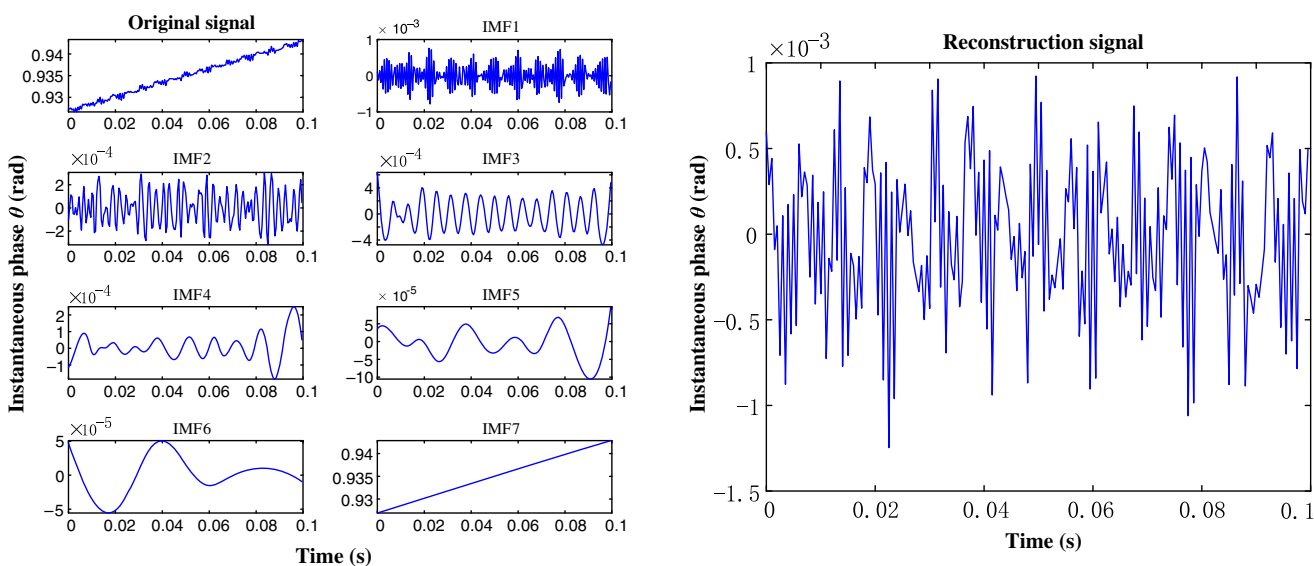

Fig. 15 Section II gear wear instantaneous phase signal decomposition and composition.

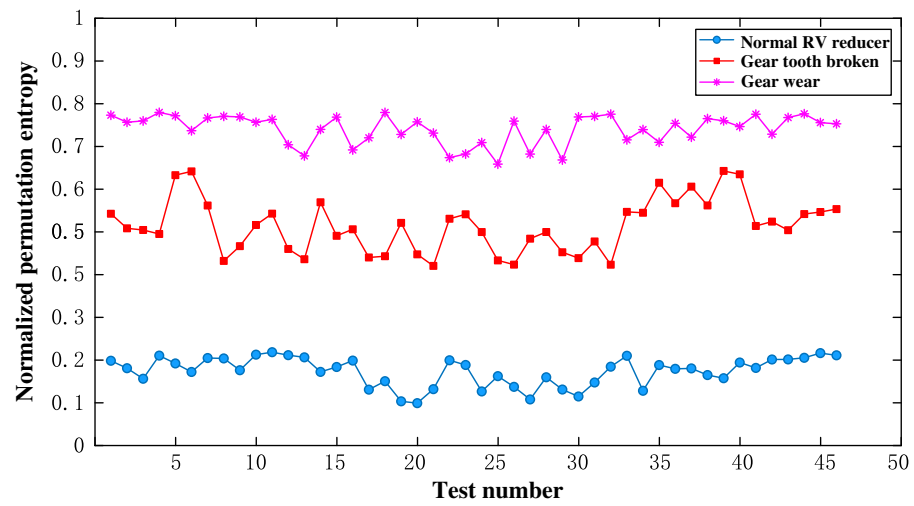

Fig. 16 Dynamic performance evaluation curve of test gear arrangement entropy.

of worn gear in section II. Then, using the adaptive permutation entropy quantitative evaluation method based on instantaneous phase information, the permutation entropy value of normal gear reconstruction signal sequence is calculated to be 0.2064 . The permutation entropy of reconstructed signal sequence of broken gear is 0.4361 . The permutation entropy of signal sequence reconstructed by worn gear is 0.6780 .

To further verify the feasibility and reliability of the detection method and quantitative evaluation method proposed in this paper, batch tests were carried out under different working conditions and different gears were replaced, and the permutation entropy value was calculated. Figure 16 shows the standard permutation entropy curve of batch test gear. The calculation results of gear permutation entropy in batch tests are shown in Table 1. It can be seen from the figure and table that using permutation entropy index can effectively distinguish gears with different quality problems.

Table 1 Test gear arrangement entropy dynamic performance evaluation results.

\begin{tabular}{lc}
\hline \hline RV reducer fault type & Permutation entropy distribution \\
\hline Normal RV reducer & 0.0000 to 0.2185 \\
RV reducer gear tooth broken & 0.4205 to 0.6428 \\
RV reducer gear wear & 0.6587 to 0.7797 \\
\hline \hline
\end{tabular}




\section{Conclusion}

In this paper, RV-20E-121 RV reducer industrial robot shoulder core components were the research object, and the spur gear setup's broken teeth and tooth surface wear were two faults. To obtain the fault signal of the RV spur gear accurately and quickly, an instantaneous phase detection technology based on an eccentric modulation principle was proposed, which used laser displacement sensor and eccentric sleeve to accurately obtain the instantaneous phase signal containing the fault characteristics of spur gear. At the same time, a quantitative evaluation method based on adaptive permutation entropy is proposed, which can classify RV reducer basic components with different quality problems quickly and effectively. A test platform for fault diagnosis of the RV reducer basic components was developed to verify the validity and reliability of detection technology and quantitative evaluation technology. The test results show that the platform can effectively judge whether there are faults and performance defects of the RV reducer spur gear.

\section{Acknowledgments}

This work is supported by the National Key Research and Development Program (Grant Nos. SQ2020YFB130256 and 2020YFB1314203) and the National Natural Science Foundation of China (Grant No. 51705003). The authors would like to thank the anonymous reviewers for their insightful comments and useful suggestions that help to improve the quality of this work.

\section{References}

1. T. Bi et al., "Research on the influence of laser-tuning characteristics on all-fiber distributed Bragg reflector self-mixing rangefinder," Opt. Eng. 57(5), 051505 (2018).

2. X. Zhang et al., "Dual-differential laser Doppler angular vibrometer using a ring laser," Opt. Eng. 55(10), 106107 (2016).

3. E. Bohkman et al., "Implementation of the dynamic laser goniometer for noncontact measurement of angular movement," Opt. Eng. 55(7), 074104 (2016).

4. F. Deng et al., "Life calculation of angular contact ball bearings for industrial robot RV reducer," Ind. Lubric. Tribol. 71(6), 826-831 (2019).

5. S. Wang et al., "Study on torsional vibration of RV reducer based on time-varying stiffness," J. Vibr. Eng. Technol. 9(1), 73-84 (2021).

6. H. Xu et al., "Optimal measurement speed and its determination method in the transmission precision evaluation of precision reducers," Appl. Sci. 9(10), 2146 (2019).

7. Z. Zhang et al., "Analysis of mixed lubrication of RV reducer turning arm roller bearing," Ind. Lubric. Tribol. 70(3), 1-20 (2017).

8. R. Wang et al., "Meshing efficiency analysis of modified cycloidal gear used in the RV reducer," Tribol. Trans. 62(3), 337-349 (2019).

9. Y. Yang et al., "A modelling approach for kinematic equivalent mechanism and rotational transmission error of RV reducer," Mech. Mach. Theory 163, 104384 (2021).

10. L. Chen et al., "Application of nonlinear output frequency response functions and deep learning to RV reducer fault diagnosis," IEEE Trans. Instrum. Meas. 70, 1-14 (2021).

11. A. Rohan, I. Raouf, and H. S. Kim, "Rotate vector (RV) reducer fault detection and diagnosis system: towards component level Prognostics and Health Management (PHM),' Sensors 20(23), 6845 (2020).

12. W. Yu et al., "Intelligent fault diagnosis of planetary gearbox based on refined composite hierarchical fuzzy entropy and random forest," ISA Trans. 109, 340-351 (2021).

13. X. Yu, Z. Feng, and M. Liang, "Analytical vibration signal model and signature analysis in resonance region for planetary gearbox fault diagnosis," J. Sound Vibr. 498(1-2), 115962 (2021).

14. K. Jiang et al., "In-process quality inspection of rolling element bearings based on the measurement of microelastic deformation of outer ring," Shock Vibr. 2019, 1-12 (2019).

15. J. Yu and X. Zhou, "One-dimensional residual convolutional autoencoder based feature learning for gearbox fault diagnosis," IEEE Trans. Ind. Inf. 16(10), 6347-6358 (2020). 
16. H. Pan et al., "Nonlinear sparse mode decomposition and its application in planetary gearbox fault diagnosis," Mech. Mach. Theory 155, 104082 (2020).

17. S. Schmidt, S. Heyns, and K. Gr Yllias, "An informative frequency band identification framework for gearbox fault diagnosis under time-varying operating conditions," Mech. Syst. Signal Process. 158, 107771 (2021).

18. K. Jiang et al., "Transient waveform matching based on ascending multi-wavelets for diagnostics and prognostics of bearing deterioration," ISA Trans. 120, 330-341 (2022).

Lianghe Li received his BS degree from Anhui University of Science and Technology, Huainan, China, in 2019, where he is currently pursuing his MS degree in mechanical engineering. His research interests include machine vision, signal processing, and fault diagnosis.

Kuosheng Jiang received his $\mathrm{PhD}$ from the State Key Laboratory for Manufacturing System Engineering, School of Mechanical Engineering, Xi'an Jiaotong University, Xi'an, China, in 2016. He is currently at Anhui University of Science and Technology, Huainan, China. His research interests include machine vision, intelligent instrument, signal processing, and fault diagnosis.

Kaisong Wang received his $\mathrm{PhD}$ from the School of Mechanical and Automotive Engineering, Hefei University of Technology in 2008. He is currently at Anhui University of Science and Technology, Huainan, China. His research interests include mechanical structure design and analysis and vehicle structure design.

Zhongyuan Mao received his BS degree from Zhejiang University of Technology in 2020, majoring in communication engineering. He is a postgraduate student at Anhui University of Science and Technology, majoring in electronic information. His research direction is intelligent instruments and machine vision.

Zaichuan Fan received his BS degree from Anhui University of Science and Technology, Huainan, China, in 2020, where he is currently pursuing an MS degree in electronic information. His research interests include signal processing and machine vision.

Jiashu Hou received her BS degree from Anhui University of Technology in 2020. Currently, she is studying for her master's degree at Anhui University of Science and Technology. Her main research interests are azimuth intelligent instruments and sensing technology. 